\title{
Identifiability and Stability of Boundaries in a Supercritical Free Surface Flow
}

\author{
Djamel-Eddine TENIOU, Rachida AIT-YAHIA, \\ and Dahbia HeRnANe \\ Laboratoire d'Analyse Mathématique et Numérique \\ des Equations aux Dérivées Partielles \\ Houari Boumédienne, Faculté de Mathématiques \\ Université des Sciences et de la Technologie \\ BP $n^{\mathrm{O}} 32$ El Alia \\ Bab-Ezzouar, Alger - Algerie. \\ dteniou@yahoo.fr rachidadjouadi@hotmail.com \\ dboukari_d@yahoo.fr
}

Received: July 27, 2006

Accepted: May 18, 2007

\begin{abstract}
In this paper, we have studied a problem of identifiability of boundaries and stability of the solutions for the direct and the inverse problem concerning a supercritical and irrotational flow of an inviscid fluid over an obstacle which lies on the bottom of a channel. The identifiability of the solution means its uniqueness when it exists. The stability is studied in the sense that for the direct problem and the inverse one, we study the variation of the obtained geometry for a little perturbation of the bottom or of the free surface. The proofs of the theorems are based on Holmgren theorem and the mean value theorem. The stability obtained is linear.
\end{abstract}

Key words: inverse problem, free boundary, identifiability, stability.

2000 Mathematics Subject Classification: 35R35, 76B07.

\section{Position of the problem}

We consider a bidimensional, irrotational and stationary flow of an ideal and incompressible fluid in a domain $\Omega$ infinite at the downstream and the upstream, with a free surface at the upper boundary and an obstacle on the bottom. We suppose that 


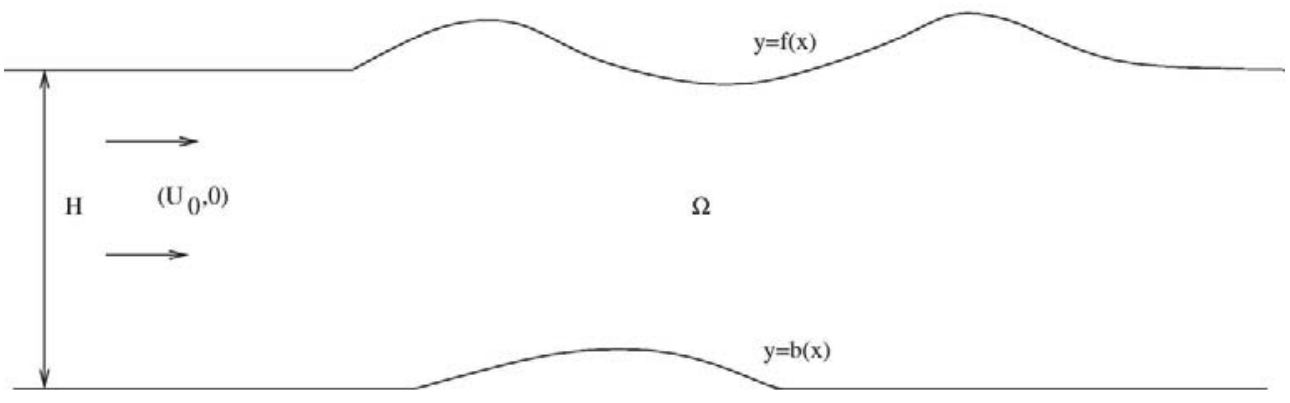

Figure 1

the flow is uniform with a velocity $U_{0}$ at the infinity, upstream and downstream of the obstacle, where the depth of the flow is denoted $H$ (see figure 1).

The bottom is flat everywhere except in a place where lies an obstacle. $y=b(x)$ and $y=f(x)$ are respectively the equations of the bottom $\Gamma_{b}$ and the free surface $\Gamma_{f}$. We denote

$$
\Omega=\left\{(x, y) \in \mathbb{R}^{2} \mid b(x)<y<f(x)\right\}
$$

We suppose that the functions $b$ and $f$ are of the class $C^{2}$ on $\mathbb{R}$. (in the case of a small height of the obstacle, $f$ belongs to $C^{2}$ because $b$ belongs to $C^{2}$, see [4]).

In [4], it has been established existence and uniqueness of the free surface for a small obstacle. In this paper, our aim is to generalize the uniqueness for obstacles without condition on their height and to establish the identifiability of the obstacle when the free surface is known.

The irrotationality of the flow and the incompressibility of the fluid permits us to introduce an harmonic stream function $\psi$ in $\Omega$. We give the relations verified by $\psi$. The bottom and the free surface of $\Omega$ are streamlines so $\psi$ is constant on $\Gamma_{b}$ and $\Gamma_{f}$. We choose $\psi=0$ on the bottom and equal to the rate of flow on the free surface. The flow being uniform at the infinity upstream and downstream of the obstacle, the function $\psi$ equals $y$ when $x$ tends to $\pm \infty$. In addition, we have an equilibrium condition on the free surface, called the Bernoulli equation, which expresses the continuity of the pressure across the free surface. It is given by

$$
\frac{\rho}{2}|\nabla \psi|^{2}+\rho g y=C^{t e} \quad \text { on } y=f(x)
$$

where $\rho$ is the density of the fluid and $g$ the gravity. (For example, see [4] for details.) The constant which appears in the relation (1) will be evaluated later. Using the values of $U_{0}$ and $H$, we nondimensionalize the equations verified by $\psi$. Then it appears a number $F=\frac{U_{0}}{\sqrt{g H}}$, called the Froude number which characterizes the flow. 
The flow is said supercritical when $F>1$ and subcritical when $F<1$. In this paper, we will focus ourselves on the supercritical case.

The Bernoulli equation becomes

$$
\frac{F^{2}}{2}|\nabla \psi|^{2}+y=C^{t e} \quad \text { on } y=f(x) .
$$

$\psi$ being constant on the free surface, the tangential derivative of $\psi$ is equal to zero on this surface. The equation (2) takes the new form

$$
\frac{F^{2}}{2}\left(\frac{\partial \psi}{\partial n}\right)^{2}+y=C^{t e} \quad \text { on } y=f(x)
$$

where $\frac{\partial \psi}{\partial n}$ is the normal derivative of $\psi$ at the free surface. The constant in equation (3) is equal to $\frac{F^{2}}{2}+1$. Indeed at the infinity upstream of the obstacle, we have

$$
\lim _{x \rightarrow-\infty} \frac{\partial \psi}{\partial n}=\lim _{x \rightarrow-\infty} \frac{\partial \psi}{\partial y}=1 \quad \text { and } \quad y=1,
$$

and so

$$
\frac{F^{2}}{2}+1=C^{t e}
$$

The equation (3) becomes

$$
\left(\frac{\partial \psi}{\partial n}\right)^{2}=c_{1}-c_{2} y \quad \text { on } y=f(x)
$$

where

$$
c_{1}=1+\frac{2}{F^{2}} \text { and } c_{2}=\frac{2}{F^{2}} .
$$

We note that if a solution exists, it necessarily verifies: $y<\frac{c_{1}}{c_{2}}$. Then we obtain

$$
\frac{\partial \psi}{\partial n}= \pm \sqrt{c_{1}-c_{2} y} \quad \text { on } y=f(x) .
$$

In fact we have

$$
\frac{\partial \psi}{\partial n}=+\sqrt{c_{1}-c_{2} y} \quad \text { on } y=f(x) .
$$

Indeed

$$
\frac{\partial \psi}{\partial y}=a(x, y) \frac{\partial \psi}{\partial n} \quad \text { on } \Gamma_{f} .
$$

The direction of the flow is towards the positive $x$. Then the horizontal component of the velocity at each point on any streamline is positive. The velocity is equal to curl $\psi$ then $\frac{\partial \psi}{\partial y}(x, y)>0$. Hence $\psi$ is an increasing function of $y$. Then

$$
a(x, y) \frac{\partial \psi}{\partial n}>0 \quad \text { on } \Gamma_{f} .
$$


On the other hand, at the infinity upstream of the obstacle we have

$$
\frac{\partial \psi}{\partial n}=\frac{\partial \psi}{\partial y} \quad \text { on } \Gamma_{f}
$$

Then

$$
\lim _{x \rightarrow-\infty} a(x, y(x))=1 \quad \text { on } \Gamma_{f} .
$$

From (6) and (7) we have that $a(x, y)>0$ on $y=f(x)$. We conclude by (5) that

$$
\frac{\partial \psi}{\partial n}>0 \quad \text { on } y=f(x)
$$

Finally $\psi$ verifies the following problem:

$$
\begin{cases}\Delta \psi=0 & \text { in } \Omega, \\ \psi=0 & \text { on } y=b(x), \\ \psi=1 & \text { on } y=f(x), \\ \frac{\partial \psi}{\partial n}=\sqrt{c_{1}-c_{2} y} & \text { on } y=f(x),\end{cases}
$$

where $c_{1}$ and $c_{2}$ are defined in the relation (4) and

$$
\lim _{x \rightarrow \pm \infty} \psi(x, y)=y
$$

We call (8), (9) a direct problem if $b$ is known and $f$ is to be determined. It will be called an inverse problem in the opposite case. ie., $f$ is known (or at least a part of $f$ ) and $b$ is to be identified. In this work, we will consider these two aspects of the problem: the direct problem and the inverse problem. In the direct problem, for a given obstacle, we prove the uniqueness of the solution if it exists. Concerning the inverse problem, we consider two domains $\Omega_{1}$ and $\Omega_{2}$ with bottoms $\Gamma_{b_{1}}$ and $\Gamma_{b_{2}}$ described by $y=b_{1}(x)$ and $y=b_{2}(x)$, with free surfaces $\Gamma_{H_{1}}$ and $\Gamma_{H_{2}}$ described by $y=f_{1}(x)$ and $y=f_{2}(x)$ which coincide on a part $\Gamma_{C}$. Then $b_{1}(x)=b_{2}(x)$. Using the result of the direct problem, we conclude that $f_{1}(x)=f_{2}(x)$. Hence $\Omega_{1}=\Omega_{2}$. Later, we will study the stability of the bottom of the domain and of the free surface. We note that some authors have studied the identifiability and the stability of boundaries for inverse problems (see for example [2]). For small obstacles, the existence and uniqueness of the solution of the direct problem is done in [4] where we have used the implicit function theorem. The stability of this solution is deduced from this theorem.

\section{Identifiability of the boundaries}

\subsection{Identifiability of the free surface}

We have the following result: 

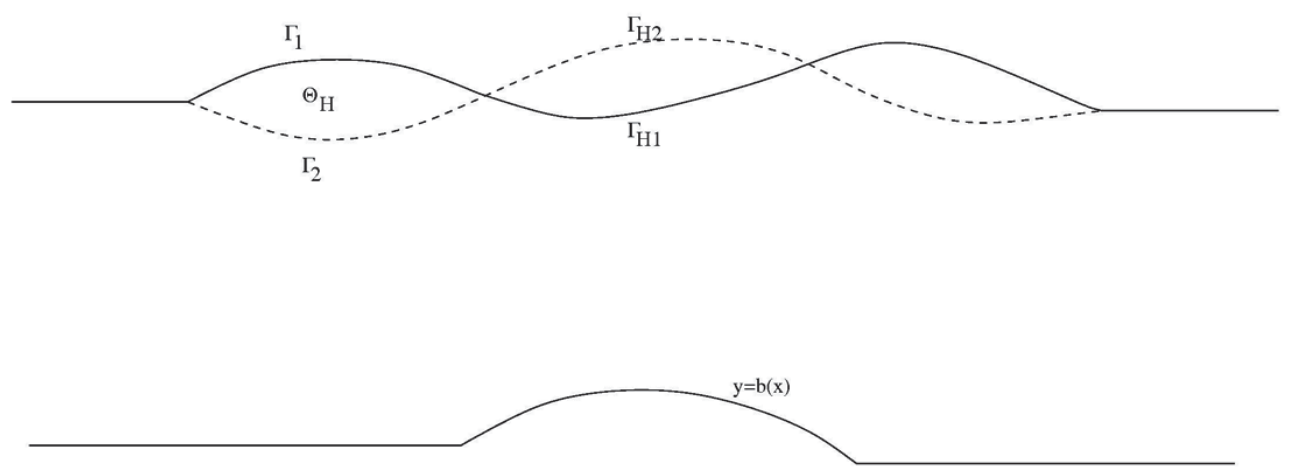

Figure 2

Theorem 2.1. For an obstacle given by a function $y=b(x)$ of class $C^{2}$, lying on the bottom of a channel, it corresponds at most one free surface $f$ of class $C^{2}$ such that $\lim _{x \rightarrow \pm \infty} f(x)=1$.

Proof. We denote by $y=b(x)$ the equation of the bottom of $\Omega$ and we suppose the existence of two free surfaces $\Gamma_{H_{1}}$ and $\Gamma_{H_{2}}$ described respectively by $y=f_{1}(x)$ and $y=f_{2}(x)$ (see figure 2).

We put

$$
\Omega_{i}=\left\{(x, y) \in \mathbb{R}^{2} \mid b(x)<y<f_{i}(x)\right\} .
$$

The stream function $\psi_{i}$ defined on $\Omega_{i}$ verifies

$$
\begin{cases}\Delta \psi_{i}=0 & \text { in } \Omega_{i}, \\ \psi_{i}=0 & \text { on } y=b(x), \\ \psi_{i}=1 & \text { on } y=f_{i}(x), \\ \lim _{x \rightarrow \pm \infty} \psi_{i}(x, y)=y . & \end{cases}
$$

On the other hand, the Bernoulli condition is verified on any streamline of the domain $\Omega_{i}$. This condition, written on the bottom, is given by

$$
\frac{F^{2}}{2}\left|\nabla \psi_{i}\right|^{2}+b(x)=\frac{F^{2}}{2} .
$$

It may be written

$$
\frac{\partial \psi_{i}}{\partial n}=\sqrt{1-\frac{2}{F^{2}} b(x)} .
$$

Let $\Theta=\left(\Omega_{1} \cup \Omega_{2}\right) \backslash\left(\overline{\Omega_{1} \cap \Omega_{2}}\right)$. Suppose that $\Theta \neq \emptyset$. Let $\Theta_{H}$ a connected component of $\Theta$. Suppose for example that $\Theta_{H} \subset \Omega_{1} \backslash\left(\overline{\Omega_{1} \cap \Omega_{2}}\right)$. The boundary of $\Theta_{H}$ is 


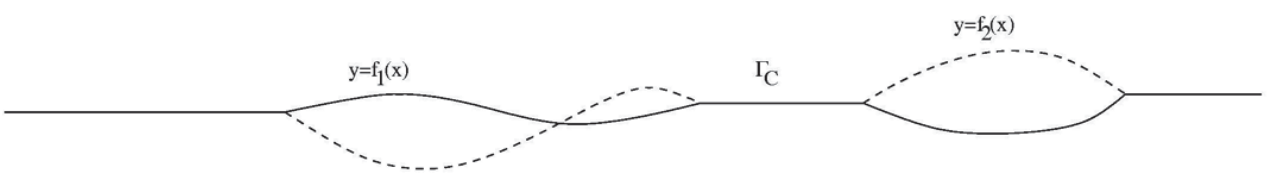

$\Omega$

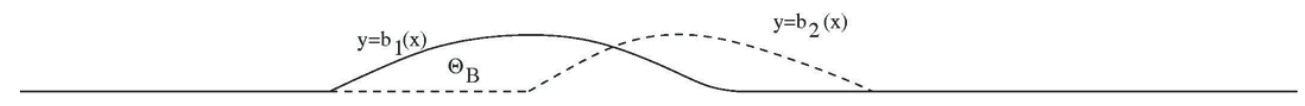

Figure 3

composed by a part of $\Gamma_{H_{1}}$ which we denote $\Gamma_{1}$ and a part of $\Gamma_{H_{2}}$ which we denote $\Gamma_{2}$ (see figure 2).

The function $\psi=\psi_{1}-\psi_{2}$ verifies the following system:

$$
\begin{cases}\Delta \psi=0 & \text { in } \Omega_{1} \cap \Omega_{2}, \\ \psi=0 & \text { on } y=b(x), \\ \frac{\partial \psi}{\partial n}=0 & \text { on } y=b(x), \\ \lim _{x \rightarrow \pm \infty} \psi(x, y)=0 . & \end{cases}
$$

We apply the Holmgren theorem [6] to deduce that $\psi=0$ in $\Omega_{1} \cap \Omega_{2}$, so $\psi_{1}=\psi_{2}$ on the boundary of $\Omega_{1} \cap \Omega_{2}$, particularly on $\Gamma_{2}$. In fact $\psi_{2}=1$ on $\Gamma_{2}$, and then $\psi_{1}=1$ on $\Gamma_{2}$. This is a contradiction with the increasing of the function $\psi_{1}$ with respect to $y$ (because $\psi_{1}$ can not take the value one on $\Gamma_{1}$ and on $\Gamma_{2}$ ). We conclude that $\Theta=\emptyset$, and then $f_{1}(x)=f_{2}(x)$.

\subsection{Identifiability of the bottom}

We consider $\Omega_{1}$ and $\Omega_{2}$ two possible domains with bottoms described respectively by $y=b_{1}(x)$ and $y=b_{2}(x)$ and free surfaces described respectively by $y=f_{1}(x)$ and $y=f_{2}(x)$ of class $C^{2}$, having a common part $\Gamma_{C}$ (see figure 3 ) where $\Gamma_{C}$ have a non empty interior. We want to prove the uniqueness of the bottom for a given part $\Gamma_{C}$ of the upper free boundary.

We denote these two domains by

$$
\Omega_{i}=\left\{(x, y) \in \mathbb{R}^{2} \mid b_{i}(x)<y<f_{i}(x)\right\} .
$$


The stream function $\psi_{i}$ verifies in $\Omega_{i}$ the following problem:

$$
\begin{cases}\Delta \psi_{i}=0 & \text { in } \Omega_{i}, \\ \psi_{i}=0 & \text { on } y=b_{i}(x), \\ \psi_{i}=1 & \text { on } y=f_{i}(x), \\ \frac{\partial \psi_{i}}{\partial n}=\sqrt{c_{1}-c_{2} y} & \text { on } y=f_{i}(x) .\end{cases}
$$

Moreover we have $\lim _{x \rightarrow \pm \infty} \psi_{i}(x, y)=y$.

Theorem 2.2. Under the hypothesis above, we have

$$
b_{1}(x)=b_{2}(x) \quad \text { and } \quad f_{1}(x)=f_{2}(x) \quad \forall x \in \mathbb{R} .
$$

Proof. The proof is the same as the one in theorem 2.1. Let $\Theta=\left(\Omega_{1} \cup \Omega_{2}\right) \backslash\left(\overline{\Omega_{1} \cap \Omega_{2}}\right)$. Suppose that $\Theta \neq \emptyset$. Let $\Theta_{B}$ a connected component of $\Theta$.

Suppose, for example, that $\Theta_{B} \subset \Omega_{2} \backslash\left(\overline{\Omega_{1} \cap \Omega_{2}}\right)$. We denote $\Omega=\Omega_{1} \cap \Omega_{2}$ and we put $\psi=\psi_{1}-\psi_{2} \cdot \psi$ verifies the Cauchy problem

$$
\begin{cases}\Delta \psi=0 & \text { in } \Omega, \\ \psi=0 & \text { on } \Gamma_{C}, \\ \frac{\partial \psi}{\partial n}=0 & \text { on } \Gamma_{C}\end{cases}
$$

The Holmgren theorem [6] permits us to conclude that $\psi=0$ in the connected domain $\Omega$. Then

$$
\psi=0 \quad \text { on } \partial \Omega \text {. }
$$

This implies that $\psi_{1}=\psi_{2}$ on $\partial \Omega$. In particular $\psi_{1}=\psi_{2}=0$ on $\Gamma_{b_{1}}$. This is a contradiction with the fact that $\psi_{2}=0$ on $\Gamma_{b_{2}}$ and the fact that $\psi_{2}$ is an increasing character function with respect to $y$, then $\Theta=\emptyset$ and $b_{1}(x)=b_{2}(x)$.

The identifiability result of the direct problem (theorem 1) permits us to conclude that $f_{1}(x)=f_{2}(x) \forall x \in \mathbb{R}$ and then $\Omega_{1}=\Omega_{2}$.

Remark 2.3. We can formulate the last theorem in this way:

Let $b_{1}$ and $b_{2}$ two possible bottoms to identify. Let $\psi_{i}$ the solution of the problem (10). Then

$$
\frac{\partial \psi_{1}}{\partial n}=\frac{\partial \psi_{2}}{\partial n} \quad \text { on } \Gamma_{C} \quad \text { implies } \quad b_{1}=b_{2}
$$

\section{Stability of the boundaries of the domain}

First, we give some notations, which will be useful from now on. 


\subsection{Some notations}

The fluid domain $\Omega$ given by a figure at the first section can be written as

$$
\Omega_{b}^{\gamma}=\left\{(x, y) \in \mathbb{R}^{2} \mid-\infty<x<+\infty, \quad b(x)<y<1+\gamma(x)\right\},
$$

where $b$ is the function describing the bottom and $\gamma$ is the perturbation of the horizontal surface $y=1$. Using a change of variables,

$$
\left\{\begin{array}{l}
\tilde{x}=x, \\
\tilde{y}=\frac{y-b(x)}{1+\gamma(x)-b(x)},
\end{array}\right.
$$

the domain $\Omega_{b}^{\gamma}$ becomes the rectangle

$$
\Omega_{0}^{0}=\left\{(x, y) \in \mathbb{R}^{2} \mid-\infty<x<+\infty, 0<y<1\right\}
$$

and the relation (2) takes the form $T(b, \gamma)=0$, where

$$
\begin{aligned}
T(b, \gamma)=\gamma-\frac{F^{2}}{2}\left\{\left[\frac{\partial \tilde{\psi}}{\partial \tilde{x}}(\tilde{x}, 1)\right.\right. & \left.-\frac{\gamma^{\prime}}{(1+\gamma-b)^{2}} \frac{\partial \tilde{\psi}}{\partial \tilde{y}}(\tilde{x}, 1)\right]^{2} \\
& \left.+\frac{1}{(1+\gamma-b)^{2}}\left(\frac{\partial \tilde{\psi}}{\partial \tilde{y}}(\tilde{x}, 1)\right)^{2}\right\}-\frac{F^{2}}{1+\gamma-b} \frac{\partial \tilde{\psi}}{\partial \tilde{y}}(\tilde{x}, 1)
\end{aligned}
$$

and $\tilde{\psi}$ verifies the system

$$
\begin{cases}\Delta \tilde{\psi}+\mathcal{P}_{b}^{\gamma} \tilde{\psi}=0 & \text { in } \Omega_{0}^{0} \\ \tilde{\psi}(\tilde{x}, 0)=-b(\tilde{x}), & \tilde{x} \in \mathbb{R} \\ \tilde{\psi}(\tilde{x}, 1)=-\gamma(\tilde{x}), & \tilde{x} \in \mathbb{R}\end{cases}
$$

$\mathcal{P}_{b}^{\gamma}$ is defined by

$$
\begin{aligned}
\mathcal{P}_{b}^{\gamma} & =a_{1} \frac{\partial^{2}}{\partial \tilde{x} \partial \tilde{y}}+a_{2} \frac{\partial^{2}}{\partial \tilde{y}^{2}}+a_{3} \frac{\partial}{\partial \tilde{y}}, \\
a_{1} & =2 \frac{\tilde{y}\left(b^{\prime}-\gamma^{\prime}\right)-b^{\prime}}{1+\gamma-b}, \\
a_{2} & =\left(\frac{a_{1}}{2}\right)^{2}-1+\frac{1}{(1+\gamma-b)^{2}}, \\
a_{3} & =\frac{-1}{1+\gamma-b}\left[b^{\prime \prime}+\tilde{y}\left(\gamma^{\prime \prime}-b^{\prime \prime}\right)\right]+\frac{2}{(1+\gamma-b)^{2}}\left(\gamma^{\prime}-b^{\prime}\right)\left[b^{\prime}+\tilde{y}\left(\gamma^{\prime}-b^{\prime}\right)\right] .
\end{aligned}
$$

$b$ and $\gamma$ are in the space

$$
B_{c}^{2, \lambda}(\mathbb{R})=\left\{v \in C^{2, \lambda}(\mathbb{R})\left|\sum_{0 \leq k \leq 2} \sup _{x \in \mathbb{R}} e^{c|x|}\right| D_{x}^{k} v(x) \mid<\infty\right\},
$$


where $0<\lambda<1$ and $c>0$. It has been proved in [4] that for a given small $b$ in $B_{c}^{2, \lambda}(\mathbb{R})$, there exists one and only one $\gamma$ in the same space such that $(b, \gamma)$ verifies $T(b, \gamma)=0$ with $\tilde{\psi}$ solution of $(12)$.

\subsection{Stability of the lower boundary of the domain}

In this section, our aim is to establish a stability result of the bottom of the domain with respect to the observed free surface. This stability expresses the continuity of the bottom configuration with respect to the data on $\Gamma_{C}$. For this study, we define a set of admissible bottoms as follows:

$$
\Gamma_{\mathrm{ad}}=\left\{(x, b(x)) \mid b \in C^{2, \lambda}(\mathbb{R}), \operatorname{supp} b \subset[-M, M], M>0\right\}
$$

where $\operatorname{supp} b$ is the compact support of $b$.

We define on $\Gamma_{\mathrm{ad}}$ the distance

$$
d\left(\Gamma_{1}, \Gamma_{2}\right)=\left\|b_{1}(x)-b_{2}(x)\right\|_{C^{2, \lambda}(\mathbb{R})} .
$$

Note that $\left(\Gamma_{\mathrm{ad}}, d\right)$ is a compact metric space.

Theorem 3.1. Let $\Gamma_{b_{n}}$ a sequence of elements of $\Gamma_{\mathrm{ad}}$. Let $\Phi_{n}$ the trace of the normal derivative of the solution of the problem (8) defined on $\Omega_{n}$ with bottom $\Gamma_{b_{n}}$ and free surface $\Gamma_{f_{n}}$. Let $\Phi$ the trace of the normal derivative of the solution of (8) defined on $\Omega$ with bottom $\Gamma_{b}$ and free surface $\Gamma_{f}$. Then we have

$$
\Phi_{n} \rightarrow \Phi \text { in } B_{c}^{1, \lambda}(\mathbb{R}) \quad \Longrightarrow \quad \Gamma_{b_{n}} \rightarrow \Gamma_{b} \text { in } \Gamma_{\mathrm{ad}}
$$

Proof. To prove this theorem, we begin by showing the continuity of the application

$$
\begin{aligned}
& \Gamma_{\mathrm{ad}} \longrightarrow B_{c}^{1, \lambda}(\mathbb{R}) \\
& \Gamma_{b} \longmapsto \frac{\partial \psi}{\partial n} .
\end{aligned}
$$

Indeed, $\Gamma_{\mathrm{ad}}$ is isomorph to the following space:

$$
B=\left\{b \in C^{2, \lambda}(\mathbb{R}) \mid b \text { with a compact support included in }[-M, M]\right\} .
$$

and we have

$$
\begin{array}{ccccc}
B & \longleftrightarrow & B_{c}^{2, \lambda}(\mathbb{R}) & \longleftrightarrow & B_{c}^{1, \lambda}(\mathbb{R}) \\
b & \longmapsto & b & \longmapsto & \frac{\partial \psi}{\partial n} .
\end{array}
$$

So we have the continuity of the application given by (14). $\Gamma_{\text {ad }}$ being compact, we can extract a subsequence noted again $\Gamma_{b_{n}}$ that tends to $\Gamma_{b_{1}}$. Thanks to the continuity of the application $\Gamma \mapsto \Phi=\frac{\partial \psi}{\partial n}$, we obtain $\Phi_{n} \rightarrow \Phi_{1}$, which is the trace of the normal derivative of the solution of the problem (8) defined on $\Omega_{b_{1}}$ with free surface $\Gamma_{f}$. The uniqueness of the limit allows to $\Phi_{1}=\Phi$; in fact, if $\Phi_{1}=\Phi$, we have $\frac{\partial \psi_{1}}{\partial n}=\frac{\partial \psi}{\partial n}$ on $\Gamma_{C}$, and then $b_{1}=b$ (see remark 2.3). 


\subsection{Stability of the free boundary for a small perturbation of the linear lower boundary}

In this section our aim is to study the variation of the free surface with respect to the variation of the flat bottom $\Gamma_{0}(y=0)$. Here we introduce some hypothesis which allows us the establish the stability result. We denote the initial domain by $\Omega_{0}^{0}$ and let $b_{h}$ a sequence of bottoms obtained by deforming the flat bottom. Let $b_{h}$ be

$$
b_{h}(x)=h \Theta(x),
$$

where $\Theta$ is in $C^{2, \lambda}(\mathbb{R})$ with a compact support and $\Theta(x) \geq 0$. We have the following result:

Theorem 3.2. Under the hypothesis and notations above, we have

$$
\lim _{h \rightarrow 0}\left\|\frac{y_{h}-y_{0}}{h}\right\|_{B_{c}^{2, \lambda}(\mathbb{R})} \text { exists and is strictly positive, }
$$

where $y_{h}=1+\gamma_{h}$ and $y_{0}=1$ are the free surfaces corresponding respectively to the bottoms given by $y=b_{h}(x)$ and $y=0$.

Remark 3.3. We recall that, for $F>1, y_{0}=1$ is the unique free surface corresponding to the flat bottom $(y=0)$ but, for $F<1, y_{0}=1$ is not unique (see $[3,5]$ ).

Proof. We deduce from [4] that, for $h$ small enough, there exists a $C^{1}$ function $g$ defined in a neighborhood of zero such that

$$
y_{h}=1+g\left(b_{h}\right) \quad \text { and } \quad y_{0}=1+g(0),
$$

where $g(0)=0$ and $b_{h}$ is in a neighborhood of zero.

We have

$$
\lim _{h \rightarrow 0}\left\|\frac{y_{h}-y_{0}}{h}\right\|_{B_{c}^{2, \lambda}(\mathbb{R})}=\lim _{h \rightarrow 0}\left\|\frac{g\left(b_{h}\right)-g(0)}{h}\right\|_{B_{c}^{2, \lambda}(\mathbb{R})} .
$$

Applying the mean value theorem, we can write

$$
\left.\lim _{h \rightarrow 0}\left\|\frac{y_{h}-y_{0}}{h}\right\|_{B_{c}^{2, \lambda}(\mathbb{R})}=\lim _{h \rightarrow 0}\left\|\frac{g^{\prime}\left(c_{h}\right)\left(b_{h}\right)}{h}\right\|_{B_{c}^{2, \lambda}(\mathbb{R})} \quad \text { where } c_{h} \in\right] 0, b_{h}[,
$$

and $g^{\prime}\left(c_{h}\right)$ is a linear application from $B_{c}^{2, \lambda}(\mathbb{R})$ into itself. Because

$$
\lim _{h \rightarrow 0} \frac{b_{h}-0}{h}=\Theta(x) \quad \text { in } B_{c}^{2, \lambda}(\mathbb{R}) \quad \text { and } \quad \lim _{h \rightarrow 0} g^{\prime}\left(c_{h}\right)=g^{\prime}(0)
$$

( $g^{\prime}$ is continuous) we deduce that

$$
\lim _{h \rightarrow 0}\left\|\frac{y_{h}-y_{0}}{h}\right\|_{B_{c}^{2, \lambda}(\mathbb{R})}=\left\|g^{\prime}(0) \cdot \Theta\right\|_{B_{c}^{2, \lambda}(\mathbb{R})} .
$$


A consequence of the implicit function theorem lets us write

$$
g^{\prime}(0)=-\left(\frac{\partial T}{\partial \gamma}(0,0)\right)^{-1} \circ\left(\frac{\partial T}{\partial b}(0,0)\right) .
$$

It has been shown in [4] that $T(b, \gamma)$ is continuously differentiable with respect to $b$ and $\gamma$ at the point $(0,0)$ and $\frac{\partial T}{\partial \gamma}(0,0)$ is injective from $B_{c}^{2, \lambda}(\mathbb{R})$ into $B_{c}^{2, \lambda}(\mathbb{R})$. $\frac{\partial T}{\partial b}(0,0)$ is also injective from $B_{c}^{2, \lambda}(\mathbb{R})$ into $B_{c}^{2, \lambda}(\mathbb{R})$ (see [1]). Then we conclude that $g^{\prime}(0)$ is injective. This implies that $\left\|g^{\prime}(0) . \Theta\right\|_{B_{c}^{2, \lambda}(\mathbb{R})} \neq 0$ because $\Theta$ is nonzero. Then we arrive to the final result:

$$
\lim _{h \rightarrow 0}\left\|\frac{y_{h}-y_{0}}{h}\right\|_{B_{c}^{2, \lambda}(\mathbb{R})}>0 \text {. }
$$

\subsection{Stability of the free boundary for a small perturbation of a general lower boundary}

From the last section, for small $h$ and $h_{1}$, we can write

and

$$
y_{h}=y_{0}+\alpha \cdot h+h^{2} R(h)
$$

$$
y_{h_{1}}=y_{0}+\alpha \cdot h_{1}+h_{1}^{2} R\left(h_{1}\right),
$$

where $\alpha \neq 0$ and $R$ is a bounded function of $h$. The following lemma gives the regularity of the function $R$ :

Lemma 3.4. $y_{h}$ is an analytical function of $h$ for $h$ small enough.

Proof. We have $y_{h}=1+\gamma_{h}$ with $\gamma_{h}=g\left(b_{h}\right)$ (see (15)), where $b_{h}=h \Theta(x)$ is an analytical function of $h$. If we prove that $\psi$ is an analytical function of $b$ and $\gamma$, then $T$, given by (11), will be an analytical function of $b$ and $\gamma$. Hence, using the implicit function theorem, we deduce that $\gamma=g(b)$ is an analytical function of $b$. Since $b_{h}$ is analytical with respect to $h$, then $\gamma_{h}$ is also analytical with respect to $h$.

Now, we verify that $\tilde{\psi}$ is analytical with respect to $b$ and $\gamma$. Indeed, $\tilde{\psi}$ verifies the system

$$
\begin{cases}\Delta \tilde{\psi}+\mathcal{P}_{b}^{\gamma} \tilde{\psi}=0 & \text { in } \Omega_{0}^{0}, \\ \tilde{\psi}(x, 0)=-b(x), & x \in \mathbb{R}, \\ \tilde{\psi}(x, 1)=-\gamma(x), & x \in \mathbb{R},\end{cases}
$$

where $\mathcal{P}_{b}^{\gamma}$ is given by the relation (13). The system (18) can be written as

$$
F(\tilde{\psi},(b, \gamma))=0
$$

with

$$
F(\tilde{\psi},(b, \gamma))=\left(F_{1}(\tilde{\psi},(b, \gamma)), \quad F_{2}(\tilde{\psi},(b, \gamma)), \quad F_{3}(\tilde{\psi},(b, \gamma))\right)
$$


where

$$
\begin{aligned}
& F_{1}(\tilde{\psi},(b, \gamma))=\Delta \tilde{\psi}+\mathcal{P}_{b}^{\gamma} \tilde{\psi}, \\
& F_{2}(\tilde{\psi},(b, \gamma))=\tilde{\psi}(\cdot, 0)+b \\
& F_{3}(\tilde{\psi},(b, \gamma))=\tilde{\psi}(\cdot, 1)+\gamma
\end{aligned}
$$

The functions $F_{1}, F_{2}$, and $F_{3}$ are defined from $B_{c}^{2, \lambda}\left(\Omega_{0}^{0}\right) \times B_{c}^{2, \lambda}(\mathbb{R}) \times B_{c}^{2, \lambda}(\mathbb{R})$ into $B_{c}^{0, \lambda}\left(\Omega_{0}^{0}\right), B_{c}^{2, \lambda}(\mathbb{R})$, and $B_{c}^{2, \lambda}(\mathbb{R})$ respectively. It is easily seen that $F_{1}, F_{2}$, and $F_{3}$ are analytical with respect to $\tilde{\psi}, b$, and $\gamma$. Applying the implicit function theorem to the equation (19), at the point $(0,(0,0))$, we conclude that $\tilde{\psi}$ is an analytical function of $b$ and $\gamma$.

Theorem 3.5. Let $h_{1}>0$ small enough. Then,

$$
\lim _{h \rightarrow h_{1}}\left\|\frac{y_{h}-y_{h_{1}}}{h-h_{1}}\right\|_{B_{c}^{2, \lambda}(\mathbb{R})} \text { exists and is strictly positive. }
$$

Proof. The equations (16) and (17) imply

$$
\left\|\frac{y_{h}-y_{h_{1}}}{h-h_{1}}\right\|_{B_{c}^{2, \lambda}(\mathbb{R})}=\left\|\alpha+\left(h+h_{1}\right) \cdot R(h)+\frac{h_{1}^{2}\left(R(h)-R\left(h_{1}\right)\right)}{h-h_{1}}\right\|_{B_{c}^{2, \lambda}(\mathbb{R})} .
$$

The mean value theorem applied to the function $R$ lets us write

$$
\left\|\frac{y_{h}-y_{h_{1}}}{h-h_{1}}\right\|_{B_{c}^{2, \lambda}(\mathbb{R})}=\left\|\alpha+\left(h+h_{1}\right) \cdot R(h)+h_{1}^{2} R^{\prime}(\xi)\right\|_{B_{c}^{2, \lambda}(\mathbb{R})},
$$

where $\xi \in] \min \left(h, h_{1}\right), \max \left(h, h_{1}\right)\left[\right.$. When $h$ tends to $h_{1}$ (for small $h$ and $h_{1}$ ) we obtain

$$
\lim _{h \rightarrow h_{1}}\left\|\frac{y_{h}-y_{h_{1}}}{h-h_{1}}\right\|_{B_{c}^{2, \lambda}(\mathbb{R})}=\left\|\alpha+2 h_{1} \cdot R\left(h_{1}\right)+h_{1}^{2} R^{\prime}\left(h_{1}\right)\right\|_{B_{c}^{2, \lambda}(\mathbb{R})} .
$$

The triangular inequality gives

$$
\lim _{h \rightarrow h_{1}}\left\|\frac{y_{h}-y_{h_{1}}}{h-h_{1}}\right\|_{B_{c}^{2, \lambda}(\mathbb{R})} \geq\|\alpha\|_{B_{c}^{2, \lambda}(\mathbb{R})}-\left|h_{1}\right| \cdot\left\|2 R\left(h_{1}\right)+h_{1} R^{\prime}\left(h_{1}\right)\right\|_{B_{c}^{2, \lambda}(\mathbb{R})} .
$$

We can choose $h_{1}$ small enough such that

$$
\left|h_{1}\right| \cdot\left\|2 R\left(h_{1}\right)+h_{1} R^{\prime}\left(h_{1}\right)\right\|_{B_{c}^{2, \lambda}(\mathbb{R})}<\frac{\|\alpha\|_{B_{c}^{2, \lambda}(\mathbb{R})}}{2} .
$$

Hence,

$$
\lim _{h \rightarrow h_{1}}\left\|\frac{y_{h}-y_{h_{1}}}{h-h_{1}}\right\|_{B_{c}^{2, \lambda}(\mathbb{R})} \geq \frac{\|\alpha\|_{B_{c}^{2, \lambda}(\mathbb{R})}}{2}>0 .
$$

Remark 3.6. The norms $\left\|y_{h}-y_{0}\right\|_{B_{c}^{2, \lambda}(\mathbb{R})}$ and $\left\|y_{h}-y_{h_{1}}\right\|_{B_{c}^{2, \lambda}(\mathbb{R})}$ are $O(h)$ and $O\left(h-h_{1}\right)$ respectively. 


\section{References}

[1] R. Ait Yahia-Djouadi, D. Hernane-Boukari, and D. Teniou, A study of the inverse of a free-surface problem, Abstr. Appl. Anal. 2 (2005), 159-171.

[2] A. Benabda, Sur quelques problèmes inverses géomètriques via des équations de conduction elliptique: Étude théorique et numérique, thèse présentée pour l'obtention du diplôme de docteur 3ème cycle, Ecole Nationale d'Ingénieurs de Tunis, 1993.

[3] M. Bouhadef, Contribution à l'étude des ondes de surface dans un canal: Application à l'écoulement au dessus d'un obstacle immergé, thèse de doctorat en Sciences Physiques, Université de Poitiers (U.E.R. Centre d'Etudes Aérodynamiques et thermiques), 1988.

[4] D. Boukari, R. Djouadi, and D. Teniou, Free surface flow over an obstacle: Theoretical study of the fluvial case, Abstr. Appl. Anal. 6 (2001), no. 7, 413-429.

[5] F. Helein, Ecoulement stationnaire dans un canal à fond presque plat, URA CNRS 853, Groupe Hydrodynamique Navale, ENSTA, Centre de l'Yvette, 91120 Palaiseau, France.

[6] F. Trèves, Basic linear partial differential equations, Pure and Applied Mathematics, vol. 62, Academic Press, New York-London, 1975. 